BULL. AUSTRAL. MATH. SOC.

VOL. 15 (1976), 155-156.

\title{
Analytic functions over Banach algebras
}

\section{R.W. Perry}

The purpose of this thesis is twofold:

(a) to examine the complete analytic function determined by a function element over a complex Banach algebra $M$; and

(b) to construct a Riemann hypersurface on which the resulting analytic configuration can be represented.

The construction is modelled on an old definition of Giorgi for analytic matrix functions in the neighbourhood of a diagonalizable matrix (see [3]). It is motivated by an observation of Gibson and Szekeres ([1]) that, in the case of $2 \times 2$ and $3 \times 3$ matrices, the non-diagonalizable function values can be obtained by a suitable limiting process.

In order to transfer the definition from matrices to general Banach algebras, it is necessary to assume that $M$ contains a maximal commutative $B^{*}$-subalgebra $D$, which takes the place of the subalgebra of diagonal matrices. The generalization leads naturally to meromorphic function elements and to the definition of points at infinity, which must be adjoined to $M$ in order that values of meromorphic functions at their poles may be assigned.

The Riemann hypersurface is constructed from an ordinary (complex) Riemann surface $R$ and the space $R(N)$ of continuous functions from the Gelfand structure space $N$ of $D$ into $R$. The space is provided with a suitable analytic structure, making essential use of a theorem of Gunning and Narasimhan [2] on the holomorphic immersion of non-compact open sets of $R$ into $C$.

The thesis also examines meromorphic differentials and their integrals

Received 17 July 1975. Thesis submitted to the University of New South Wales, May 1975. Degree approved, April 1976. Supervisor: Professor G. Szekeres. 
on the hypersurface. It turns out that the residues of differentials can only take certain special values on $M$, corresponding to conjugates of functions on $R(N)$ which take constant values on the members of a partition of $N$ into a finite number of compact subsets. For instance, if $N$ is connected all residues are scalar multiples of the identity of $M$.

\section{References}

[1] J.K. Gibson and G. Szekeres, "Analytic matrix functions", Nieva Arch. Wisk. (3) 15 (1967), 233-246.

[2] R.C. Gunning and Raghavan Narasimhan, "Immersion of open Riemann surfaces", Math. Ann. 174 (1967), 103-108.

[3] R.F. Rinehart, "The equivalence of definitions of a matric function", Amer. Math. Monthly 62 (1955), 395-414. 